\title{
Transient behavior of wind towers grounding systems under lightning strikes
}

\author{
Rodolfo Araneo $^{1} \cdot$ Salvatore Celozzi $^{1}$
}

Received: 9 July 2015/ Accepted: 6 November 2015/Published online: 9 December 2015

(c) The Author(s) 2015. This article is published with open access at Springerlink.com

\begin{abstract}
Wind turbines, because of their height and localization, are frequently subject to direct lightning strikes. Thus, the investigation on the performance of their grounding system is of paramount interest for the prediction of the potential threats either for people working in (or animals passing through) the wind farm area and for the power and control units installed in close proximity of the turbine towers. In this paper,we perform a comprensive study of the transient behavior of the earthing systems of wind turbines. The analysis is conducted in the frequency domain and an hybrid approach, based on circuit theory and Method of Moments, is adopted to fully account for resistive, inductive and capacitive couplings between elements of the ground system. The actual transient behavior is obtained by means of an Inverse Fourier Transform. The results, computed considering a typical wind turbine grounding system configuration, provide more insight on the nature of the early-time transient response of grounding systems and allow to draw up useful design guidelines.
\end{abstract}

Keywords Lightning - Wind turbines · Ground systems

\section{List of symbols}

$\begin{array}{ll}\mu_{0} & \text { Vacuum magnetic permeability } \\ \omega & \text { Angular frequency } \\ \mathbf{F} & \text { Vector of the known external current } \\ & \text { sources }\end{array}$

Rodolfo Araneo

rodolfo.araneo@uniroma1.it

Salvatore Celozzi

salvatore.celozzi@uniroma1.it

1 DIAEE-Electrical Engineering Division, "Sapienza" University of Rome, Via Eudossiana 18, 00184 Rome, Italy
$\mathbf{I}_{\mathrm{LE}}$

$\mathbf{J}_{\mathrm{LE}}$

$\mathbf{U}$

$\mathbf{V}$

$\overline{\mathbf{A}}$

$\overline{\mathbf{G}}_{\mathrm{A}}$

$\overline{\mathbf{K}}$

$\overline{\mathbf{Y}}_{\mathrm{GC}}$

$\overline{\mathbf{Y}}_{\mathrm{RL}}$

$\sigma_{i}$

$\varepsilon_{0}$

$\varepsilon_{\mathrm{r}, i}$

$\widetilde{G}_{v v}^{\mathrm{A}}, \widetilde{G}_{z z}^{\mathrm{A}}, \widetilde{G}_{z u}^{\mathrm{A}}, \widetilde{G}_{\varphi}$

$G_{\varphi}$

$I_{\mathrm{LE}, k}$

$J_{\mathrm{LE}, i}$

$k_{z n}^{p}$

$k_{0}$
Vector of the branch leakage currents Vector of the node leakage currents Vector of the average branch potentials

Vector of the nodal voltages Branch-to-node incidence matrix Magnetic vector potential dyadic Green function in the layered earth Potential-to-voltage-drop incidence matrix

Admittance matrix accounting for all the conductive and capacitive couplings among branches

Admittance matrix accounting for the internal impedance of branches and the magnetic coupling among them Electric conductivity of $i$ th ground layer

Vacuum dielectric permittivity Relative dielectric permittivity of $i$ th ground layer

Spectral domain Green's functions

Scalar electric potential Green's function in the layered earth Leakage current dispersed into the ground by the $k$-branch of the ground system

Leakage current dispersed into the ground concentrated at the $i$-node of the ground system

Propagation wavenumber of the $n$ trasmission line

Free space wavenumber 
$U_{k}$

$V_{i}$

$Z_{n}^{p}$
Indicates the propagation mode: $p=$ $e$ E-mode; $p=h$ H-mode

Potential drop over the $k$-branch of the ground system

Potential at the $i$-node of the ground system

Characteristic impedance of the $n$ trasmission line

\section{Introduction}

Wind farms are an excellent solution for the generation of clean electric power because of their reduced costs for investments and maintenance and their diffusion has been observed worldwide [1-3]. Wind turbines energy have been widely considered as one of the best choices in terms of payback time and energy conversion; thus, their installations have recently increased, interesting also those regions where lightning activity is significant and consequent damages may very likely occur $[4,5]$. Wind turbines are candidate victims for cloud-to-ground lightning mainly due to their special shape and complexity of apparatus: moreover, they are often located in isolated locations, at hill or mountain altitudes, where the high values of the keraunic level increase the probability of damage. Relevant statistics indicates that fatal damages in blades, generators and especially in control circuits are caused either by direct lightning strokes to wind turbines or transferred overvoltages from nearby fault locations [6].

Grounding systems of wind turbines are designed to prevent overvoltages and potential gradients that could damage parts of the wind turbine and to reduce human hazards. However, the design of the grounding system calls for the thorough analysis of the following peculiarities:

- the grounding system of a wind turbine is generally much smaller than those of buildings with equal height;

- grounding systems of turbines in wind farms are electrically interconnected;

- the lightning protection level for a wind turbine is much higher than that of a normal building having an equivalent foundation;

- generally poor grounding conditions are observed, i.e., high values of soil resistivity, ranging between 100 and $2000 \Omega \mathrm{m}[7,8]$.

The hazard and the risk are strictly dependent upon either values and duration of the transient phenomenon; thus, the grounding systems behavior of actual wind turbines has to be analyzed in the time domain. In addition, the transient analysis [9, 10] is important because the injection of high impulse currents into the grounding system leads to an

increase of the grounding potential during the transient state, which can occur in a danger to humans, animals, installations and equipment or in a loss of data transmitted through power or telecommunication cables [11].

The analysis is performed in the frequency domain by means of a recently proposed hybrid approach [12, 13] based on circuit theory and Method of Moments, capable to accurately account for resistive, inductive and capacitive couplings. However, in our formulation no approximations are introduced to model the interactions between elements and this improvement yields more accurate results. Then, the corresponding transient response is obtained by means of the inverse Fourier transform (IFT). Other efficient approaches have been proposed in the past, based either on analytical approximations or on numerical methods, like FD-TD, e.g. [14-17]. In the proposed method, solving accurately the Sommerfeld's integrals occurring in the Green's functions of a stratified medium, appears to be very accurate and reliable especially for the early-time response, which is responsible of the most severe solicitation for electrical and electronic apparatus and systems installed in close proximity of the wind towers.

\section{Grounding system analysis}

The system configuration is shown in Fig. 1: a wind turbine grounding system is buried and subjected to a transient current generated by the lighting strike at a certain point of blades or nacelle (the influence of the turbine tower is here neglected, since the body is in series with the considered current source). Generally, grounding systems configurations are prescribed or recommended by manufacturers: typical grounding systems are arranged in a ring shape around the tower's base and connected with the tower itself through its irons in the foundation. Low-impedance grounding system is a major prerequisite for an effective protection of wind turbines from lightning strikes. In the following, a time-harmonic variation of angular frequency $\omega$ is considered and all the quantities, unless otherwise indicated, are represented in terms of their complex phasors. Relevant time-domain features of the grounding system are obtained by means of a standard IFT.

The goal of the proposed hybrid method [18-21] is to obtain a frequency-dependent equivalent electric circuit, as done in other fields [22-25] for the grounding system where all the inductive, capacitive and conductive couplings among different conductor elements are accounted for and their analysis can be carried out in the frequency domain.

It should be highlighted that no low-frequency approximations are introduced, neither intrinsically approximate techniques are adopted, like, e.g., [26-28] based on complex images, to overcome the direct computation of 
Fig. 1 Schematic representation of a typical grounding system for wind turbines

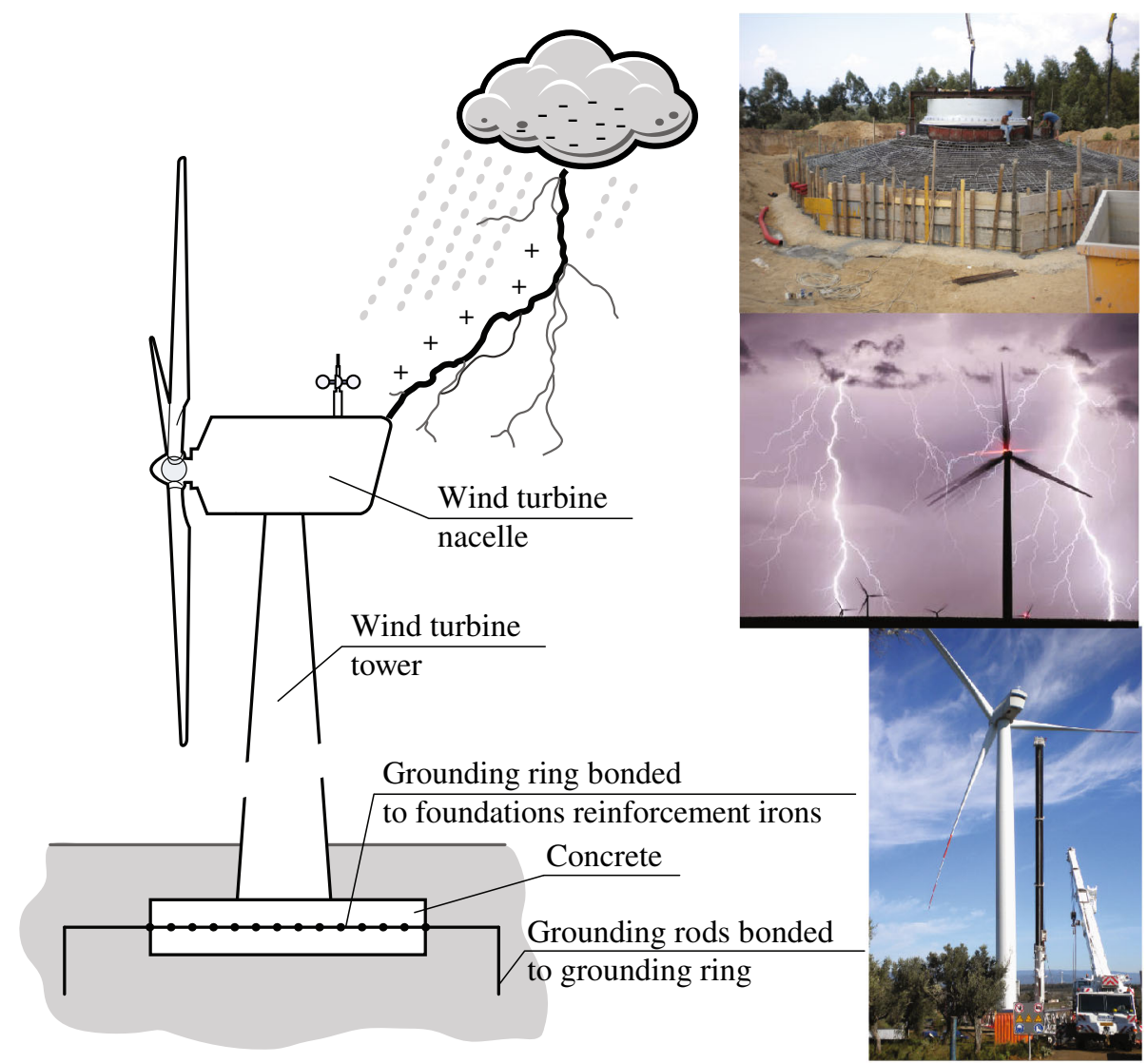

Sommerfeld's integrals arising in the Green's functions. The choice of a direct approach is motivated by the need to analyzing grounding systems even during the early time of the lightning strike when the extremely large dimensions reduce dramatically the accuracy of the currently available approximate approaches.

\section{Mathematical model}

We assume that the conductors of the grounding network are completely buried in the earth that, without loss of generality, is considered stratified in $N_{\mathrm{S}}$ planar layers. In the frequency domain, each earth layer is characterized by a complex conductivity $\bar{\sigma}_{i}=\sigma_{i}+j \omega \varepsilon_{0} \varepsilon_{\mathrm{r}, i} \quad$ (with $i=1, \ldots, N_{\mathrm{S}}$ ), where $\sigma_{i}$ is the electric conductivity, $\varepsilon_{\mathrm{r}, i}$ is the relative dielectric permittivity, $\varepsilon_{0}$ is the vacuum dielectric permittivity, and $\omega$ is the angular frequency. For all the layers the vacuum permeability, $\mu_{0}$, is assumed. Frequency dependence of ground permittivity is neglected, because of its little influence on the relevant performance in the considered frequency range of interest.

The grounding system is partitioned into $N_{\mathrm{B}}$ segments that, in the framework of the networks theory, can be studied as elemental oriented branches connecting a pair of nodes (the terminals of the branches). The discrete grounding system is assumed to have $N_{\mathrm{N}}$ nodes. Each $k$ th conductor carries a uniform longitudinal current $I_{\mathrm{LO}, k}$ flowing between the two terminal nodes whose scalar electric potentials (SEP) $V_{i}$ are defined with respect to the infinitely remote earth chosen as the zero potential reference. The introduction of the nodal voltages is necessary because at high frequencies the voltage drops along the branches are not negligible due to the electromagnetic coupling and to the increase of the internal impedance. Additionally, each branch drains a radial leakage current $I_{\mathrm{LE}, k}$ to the surrounding earth. Such a radial current is assumed to be uniform, provided that the discretization is sufficiently fine. The grounding system is energized by the injection of single-frequency sinusoidal source currents $F_{m}$ at $M$ nodes (Fig. 2).

Due to these assumptions, the SEP along each branch is not constant and varies in someway between the two voltage values at the terminal nodes. However, since the segment length is much shorter than the minimum wavelength, we can assume that the potential $U_{k}$ over the $k$ branch is constant and equal to the average of the voltages $V_{i}$ and $V_{j}$ at the two terminal nodes [29] 


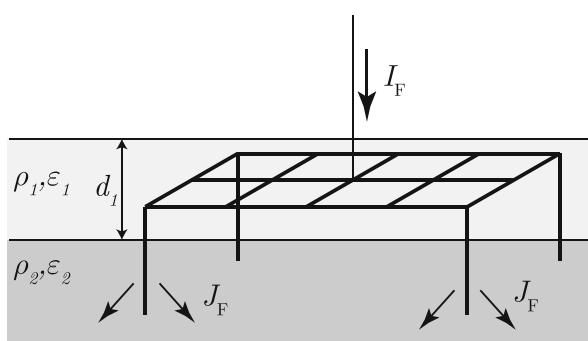

(a)

Fig. 2 Typical wind turbine grounding system arrangement (a) and section of the discretized model (b). In $\mathbf{a} I_{\mathrm{F}}$ is the fault current flowing into the earthing system and $J_{\mathrm{F}}$ is the fault current density injected into the ground. The ground is assumed stratified and each layer is

$U_{k}=\frac{V_{i}+V_{j}}{2}$.

Consequently, by applying (1) at each branch, the relationship between the $N_{\mathrm{B}} \times 1$ column vector $\mathbf{U}$ of the average SEP of segments and the $N_{\mathrm{N}} \times 1$ column vector $\mathbf{V}_{n}$ of the SEP of nodes can be expressed as

$\mathbf{U}=\overline{\mathbf{K}} \cdot \mathbf{V}$,

where $\overline{\mathbf{K}}$ is a matrix of dimensions $N_{\mathrm{B}} \times N_{\mathrm{N}}$ whose elements are

$k_{i j}=\left\{\begin{array}{ll}\frac{1}{2} & \text { if branch } i \text { is incident onto node } j \\ 0 & \text { if branch } i \text { is not incident onto node } j\end{array}\right.$.

Looking at the leakage currents and the average SEP of all branches, we found that there is a matrix relationship ensuing from the application of the Galerkin's MoM as [18]

$\mathbf{I}_{\mathrm{LE}}=\overline{\mathbf{Y}}_{\mathrm{GC}} \cdot \mathbf{U}$,

where $\mathbf{I}_{\mathrm{LE}}$ is the $N_{\mathrm{B}} \times 1$ column vector of the leakage currents and $\overline{\mathbf{Y}}_{\mathrm{GC}}$ is a $N_{\mathrm{B}} \times N_{\mathrm{B}}$ square admittance matrix accounting for all the conductive and capacitive couplings among the discretized segments, as shown in the next subsection. Subdividing each leakage current $I_{\mathrm{LE}, k}$ into two equal contributions $I_{\mathrm{LE}, k} / 2$ that are concentrated in the terminal nodes [29] (the approximation is reasonable under the assumption that the segmentation is fine with respect to the wavelength), it is possible to obtain the following relationship between the concentrated currents $\mathbf{J}_{\mathrm{LE}}$ at the nodes and the leakage currents $\mathbf{I}_{\mathrm{LE}}$ assumed uniformly distributed over each short branch

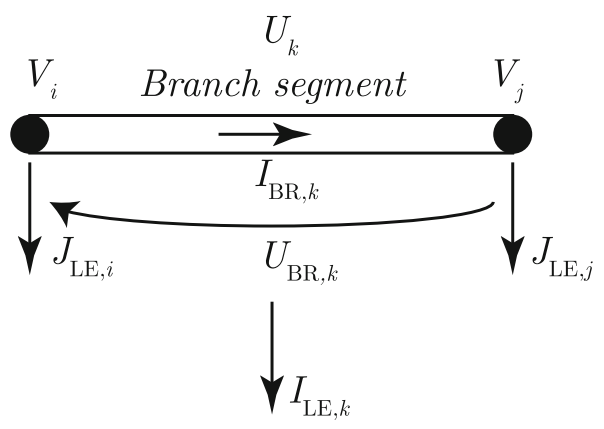

(b)

characterized by the resistivity $\rho_{i}$ and the permittivity $\varepsilon_{i}$. In $\mathbf{b} J_{\mathrm{LE}, j}$ is the leakage current at node $j, I_{\mathrm{LE}, k}$ is the leakage current dispersed by the element $k, I_{\mathrm{BR}, k}$ is the current flowing through the element $k$, and $V_{i}$ is the potential at the node $i$

$\mathbf{J}_{\mathrm{LE}}=\overline{\mathbf{K}}^{t} \cdot \mathbf{I}_{\mathrm{LE}}$

where $\mathbf{J}_{\mathrm{LE}}$ is a $N_{\mathrm{N}} \times 1$ column vector.

Under the above assumptions, the equivalent circuit can be studied making use of the conventional nodal analysis, resulting in the following equation $[12,13]$

$$
\mathbf{F}-\mathbf{J}_{\mathrm{LE}}=\left(\overline{\mathbf{A}} \cdot \overline{\mathbf{Y}}_{\mathrm{RL}} \cdot \overline{\mathbf{A}}^{t}\right) \cdot \mathbf{V},
$$

where $\mathbf{F}$ is the vector of the known external current sources, $\quad \overline{\mathbf{Y}}_{\mathrm{RL}}$ is the $N_{\mathrm{B}} \times N_{\mathrm{B}}$ square admittance matrix accounting for the internal impedance of the branches and the magnetic coupling among them, obtained through the Galerkin's MoM as described in the next paragraph, and $\overline{\mathbf{A}}$ is the $N_{\mathrm{N}} \times N_{\mathrm{B}}$ branch-to-node incidence matrix whose elements are defined as

$a_{i j}=\left\{\begin{array}{cl}+1 & \text { if branch } j \text { is directed from the node } i \\ -1 & \text { if branch } j \text { is directed toward node } i \\ 0 & \text { if branch } j \text { is not connected to node } i\end{array}\right.$.

Substituting Eqs. (2), (4), (5) into (6), the final equation is readily obtained as

$$
\mathbf{F}=\left(\overline{\mathbf{K}}^{t} \cdot \overline{\mathbf{Y}}_{\mathrm{GC}} \cdot \overline{\mathbf{K}}+\overline{\mathbf{A}} \cdot \overline{\mathbf{Y}}_{\mathrm{RL}} \cdot \overline{\mathbf{A}}^{t}\right) \cdot \mathbf{V}
$$

Once the nodal voltages are obtained, all the other relevant quantities, i.e., branch currents, leakage currents and potential on the ground, can be obtained straightforwardly.

\section{Computation of impedances}

Each branch is characterized by a lumped self-impedance. Inductive and capacitive couplings with other branches are accounted for through mutual impedances. The admittance 
Fig. 3 Equivalent TL circuit for the current and voltage wave propagation in the stratified ground

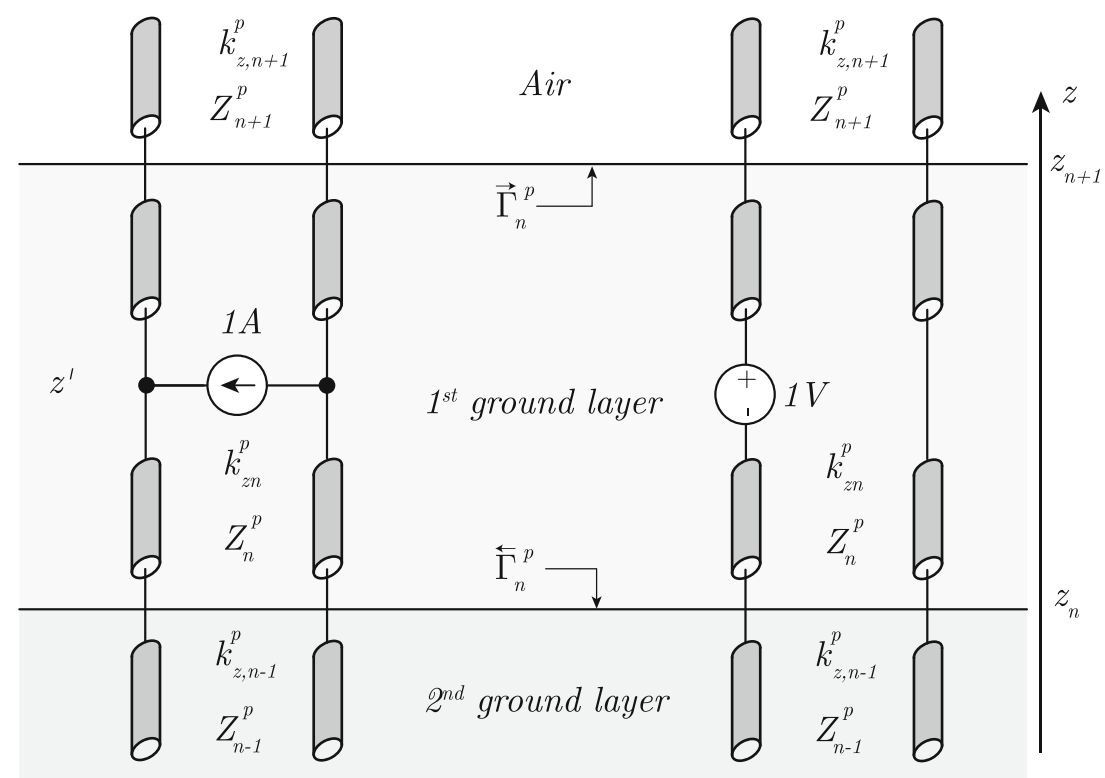

matrix $\overline{\mathbf{Y}}_{\mathrm{RL}}$ can be computed as the inverse of the impedance matrix $\overline{\mathbf{Z}}_{\mathrm{RL}}$ which account for the resistive-inductive couplings, whose elements are obtained as $[12,13]$

$Z_{\mathrm{RL}, i j}=\left\{\begin{array}{ll}Z_{i j}+j \omega L_{i j} & i=j \\ j \omega L_{i j} & i \neq j\end{array}\right.$.

In (9), $Z_{i j}$ is the internal impedance of the cylindrical conductor with radius $r_{0}$ at frequency $\omega$ that is given by

$Z_{i j}=\frac{k_{\sigma} J_{0}\left(k_{\sigma} r_{0}\right)}{2 \pi \sigma_{\mathrm{c}} r_{0} J_{1}\left(k_{\sigma} r_{0}\right)}$,

where $k_{\sigma}=\sqrt{-j \omega \mu_{\mathrm{c}} \sigma_{c}}, \sigma_{\mathrm{c}}$ and $\mu_{\mathrm{c}}$ are, respectively, the conductivity and the absolute permeability of the conductors. $J_{0}$ and $J_{1}$ are the Bessel function of the first kind and order 0 and 1 , respectively. The mutual induction between conductors $i$ and $j$ is computed as

$L_{i j}=\int_{l_{i}} \mathrm{~d} l \int_{l_{j}} \hat{\mathbf{t}} \cdot \overline{\mathbf{G}}_{\mathrm{A}}\left(\mathbf{r}, \mathbf{r}^{\prime}\right) \cdot \hat{\mathbf{t}}^{\prime} \mathrm{d} l^{\prime}$,

where $\overline{\mathbf{G}}_{\mathrm{A}}\left(\mathbf{r}, \mathbf{r}^{\prime}\right)$ is the magnetic vector potential dyadic Green function in the layered earth, $l_{i}$ and $l_{j}$ are the lengths of the $i$ th observation and $j$ th source segments with tangent unit vectors $\hat{\mathbf{t}}$ and $\hat{\mathbf{t}}^{\prime}$, respectively.

As concerns the admittance matrix $\overline{\mathbf{Y}}_{\mathrm{GC}}$, it can be computed as the inverse of the impedance matrix $\overline{\mathbf{Z}}_{\mathrm{GC}}$ which account for the conductive-capacitive couplings among elements $[12,13]$. By expanding the leakage currents in uniform zero-order basis functions $N_{i}$ defined over the $i$ th branch as $N_{i}=1 / l_{i}$ and by applying a standard Galerkin's MoM [30-32], the element $Z_{\mathrm{CG}, i j}$ is

$Z_{\mathrm{CG}, i j}=\int_{l_{i}} \frac{\mathrm{d} l}{l_{i}} \int_{l_{j}} G_{\varphi}\left(\mathbf{r}, \mathbf{r}^{\prime}\right) \frac{\mathrm{d} l^{\prime}}{l_{j}}$, where $G_{\varphi}\left(\mathbf{r}, \mathbf{r}^{\prime}\right)$ is the scalar potential Green's function in the stratified earth.

\section{Green's functions}

The dyadic magnetic Green's function in the stratified earth can be computed as follows [33]:

$\overline{\mathbf{G}}_{\mathrm{A}}\left(\rho, z, z^{\prime}\right)=\left[\begin{array}{ccc}G_{x x}^{\mathrm{A}}\left(\rho, z, z^{\prime}\right) & 0 & -G_{z x}^{\mathrm{A}}\left(\rho, z, z^{\prime}\right) \\ 0 & G_{y y}^{\mathrm{A}}\left(\rho, z, z^{\prime}\right) & -G_{z y}^{\mathrm{A}}\left(\rho, z, z^{\prime}\right) \\ G_{z x}^{\mathrm{A}}\left(\rho, z, z^{\prime}\right) & G_{z y}^{\mathrm{A}}\left(\rho, z, z^{\prime}\right) & G_{z z}^{\mathrm{A}}\left(\rho, z, z^{\prime}\right)\end{array}\right]$

with

$G_{x x}^{\mathrm{A}}\left(\rho, z, z^{\prime}\right)=G_{y y}^{\mathrm{A}}\left(\rho, z, z^{\prime}\right)=S_{0}\left\{\widetilde{G}_{v v}^{\mathrm{A}}\left(k_{\rho}, z, z^{\prime}\right)\right\}$

$G_{z z}^{\mathrm{A}}\left(\rho, z, z^{\prime}\right)=S_{0}\left\{\widetilde{G}_{z z}^{\mathrm{A}}\left(k_{\rho}, z, z^{\prime}\right)\right\}$

$G_{z z}^{\mathrm{A}}\left(\rho, z, z^{\prime}\right)=-j \cos \varphi S_{1}\left\{\widetilde{G}_{z u}^{\mathrm{A}}\left(k_{\rho}, z, z^{\prime}\right)\right\}$

$G_{z z}^{\mathrm{A}}\left(\rho, z, z^{\prime}\right)=-j \sin \varphi S_{1}\left\{\widetilde{G}_{z u}^{\mathrm{A}}\left(k_{\rho}, z, z^{\prime}\right)\right\}$,

where $S_{n}=\frac{1}{2 \pi} \int_{0}^{\infty} \tilde{f}\left(k_{\rho}\right) J_{n}\left(k_{\rho} \rho\right) k_{\rho} \mathrm{d} k_{\rho}$ is the Sommerfeld integral of order $n$.

The scalar potential Green's function is

$G_{\varphi}\left(\rho, z, z^{\prime}\right)=S_{0}\left\{\widetilde{G}_{\varphi}\left(k_{\rho}, z, z^{\prime}\right)\right\}$.

The spectral domain expressions for the elements of the Green's functions can be recast by using the transmissionline (TL) analogy described in $[34,35]$. More specifically, according to the TL notation (Fig. 3), we obtain 


$$
\begin{aligned}
& \widetilde{G}_{v v}^{\mathrm{A}}\left(z, z^{\prime}\right)=\frac{1}{j \omega \mu_{0}} V_{i}^{h}\left(z, z^{\prime}\right) \\
& \widetilde{G}_{z z}^{\mathrm{A}}\left(z, z^{\prime}\right)=\left(\frac{1}{\bar{\sigma}^{\prime}}+\frac{1}{\bar{\sigma}}\right) I_{v}^{e}\left(z, z^{\prime}\right)-\frac{j \omega \mu_{0}}{k_{\rho}^{2}}\left[I_{v}^{h}\left(z, z^{\prime}\right)-I_{v}^{e}\left(z, z^{\prime}\right)\right]
\end{aligned}
$$

$$
\widetilde{G}_{z u}\left(z, z^{\prime}\right)=\frac{1}{j k_{\rho}}\left[I_{i}^{h}\left(z, z^{\prime}\right)-I_{i}^{e}\left(z, z^{\prime}\right)\right],
$$

$$
\widetilde{G}_{\varphi}\left(z, z^{\prime}\right)=-\frac{j \omega \varepsilon_{0}}{k_{\rho}}\left[V_{i}^{h}\left(z, z^{\prime}\right)-V_{i}^{e}\left(z, z^{\prime}\right)\right]
$$

where $\mu_{0}$ and $\varepsilon_{0}$ are the free space permeability and permittivity, respectively, $k_{0}$ is the wavenumber of the free space and $k_{\rho}=\sqrt{k_{x}^{2}+k_{y}^{2}}$. In (16), $V_{i, v}^{h, e}\left(z, z^{\prime}\right)$ and $I_{i, v}^{h, e}\left(z, z^{\prime}\right)$ are, respectively, the voltage $V$ and current $I$ at the observation point $z$ on the equivalent $e$-mode (TM polarization) and $h$-mode (TE polarization) trasmission network due to a $1-\mathrm{V}$ series voltage source (subscript $v$ ) or a 1-A shunt current source (subscript $i$ ) placed at $z^{\prime}$. The trasmission networks are reported in Fig. 3: they consist of a cascade connection of uniform trasmission line sections where each section represents a layer whose boundaries are placed at $z_{n}$ and $z_{n+1}$ and is characterized by a propagation wavenumber $k_{z n}^{p}$ and characteristic impedance $Z_{n}^{p}$, with $p=e$ or $h$.

The computation of currents and voltages can be performed efficiently through the recursive computation of the generalized voltage reflection coefficients $\overleftarrow{\Gamma}_{n}^{p}$ looking to the left (towards the depth of the ground) at the $n$-interface between medium $n-1$ and medium $n$ and $\vec{\Gamma}_{n}^{p}$ (with $p=e$ or $h$ ) looking to the right (towards the air) at the $n$-interface between medium $n$ and medium $n+1$. Numerical details are reported in [34].

The computation of the Sommerfeld integrals is performed with the double exponential-type quadrature formulas recently proposed in [36]. Since the ground is lossy, the poles of the Green's functions, corresponding to guided propagation modes in the structure, move from the real axis into the forth quadrant; in addition, the Green's functions in intermediate layers, where the earthing systems are placed in practice, do not present branch points; consequently the integration can be computed straightforwardly on the real axis without deforming the Sommerfeld integral path into the first quadrant as it is usually done in order to avoid poles or branch points.

\section{Singular term}

The evaluation of the admittance and impedance matrices requires the computation of each linear wire segment comprising the earthing system. Since the basis functions for currents and charges are constant and the vector bases for current have constant direction on each linear segment, when the observation $j$ and source $i$ segments coincide, both the integrals in (11) and (12) reduce to a scalar integral of the form

$c_{\alpha} \int_{l_{i}} \mathrm{~d} l \int_{l_{j}} K\left(\mathbf{r}, \mathbf{r}^{\prime}\right) \mathrm{d} l^{\prime}$

where $c_{\alpha}$ is an appropriate coefficient and $K\left(\mathbf{r}, \mathbf{r}^{\prime}\right)$ is the corresponding kernel (e.g., the vector or scalar Green's function in the space domain). As it is well known, the Green's function in the spectral domain inside the $n$ ground layer is always composed by a first term that represents the direct ray between the source and the observation point and a second term that represents the superposition of the rays that undergo partial reflections at the upper and lower slab boundaries before reaching the field point. The first term, when is transformed back from the spectral to the spatial domain, contains a singular term that is of the form $1 /$ $R$ and that must be accurately treated when the MoM analysis is applied to wire structures in order to have nonessential (i.e., integrable) singularities.

Following the procedure presented in $[37,38]$, since the sources and the potentials about a linear tubular section present a rotational symmetry, assuming, without loss of generality, sources distributed uniformly on a cylindrical tube of constant radius $r_{0}$ centered along the $z$-axis and an observation point in cylindrical coordinates $\left(r_{0}, 0, z\right)$, the wire kernel $K\left(\mathbf{r}, \mathbf{r}^{\prime}\right)$ is defined as

$K\left(\mathbf{r}, \mathbf{r}^{\prime}\right)=\frac{1}{2 \pi} \int_{-\pi}^{\pi} \frac{\mathrm{e}^{-j k_{n} R}}{4 \pi R} \mathrm{~d} \phi^{\prime}$

where $R=\sqrt{\left(z-z^{\prime}\right)^{2}+2 r_{0}^{2}-2 \rho r_{0} \cos \phi^{\prime}}$. With simple manipulations that are not here reported for the sake of conciseness, introducing the substitution $\xi=\left(\pi-\phi^{\prime}\right) / 2$, the distance $R$ can be rewritten as $R=R_{\max } \sqrt{1-\beta^{2} \sin \alpha^{2}}$, where $R_{\max }=\sqrt{\left(z-z^{\prime}\right)^{2}+4 r_{0}^{2}}$ and $\beta=\sqrt{4 r_{0}^{2} / R_{\max }^{2}}$, and the kernel integral (18) can be expressed as

$$
K\left(\mathbf{r}, \mathbf{r}^{\prime}\right)=\frac{1}{2 \pi^{2} R_{\max }} F(\beta)+\frac{1}{2 \pi^{2}} \int_{0}^{\frac{\pi}{2}} \frac{\mathrm{e}^{-j k_{n} R}-1}{4 \pi R} \mathrm{~d} \phi^{\prime},
$$

where $F(\beta)$ is the complete elliptic integral of the first kind:

$$
F(\beta)=\int_{0}^{\frac{\pi}{2}} \frac{\mathrm{d} \phi}{\sqrt{1-\beta^{2} \sin ^{2}(\phi)}}
$$

The first term in (19) contains a logarithmic singularity as $\beta \rightarrow 1$, i.e. $z \rightarrow z^{\prime}$, while the second term is smooth and can be easily evaluated with standard Gaussian quadrature formulas. When the first term in (19) is included in the source linear integral in (17), the logarithmic singularity must be accurately integrated on the linear element, and 
this job can be carried out by using the modified quadrature rules proposed by Wandzura in [39].

\section{Lightning current parameters}

Lightning depends on a number of factors like type (i.e., positive or negative discharges), first or subsequent strokes, direction, channel shape and so forth. Each feature is characterized by its uncertainty; the phenomenon is typically analyzed on a statistical basis, corroborated by means of extensive measurements collected in instrumented towers [40-43] or in artificially-initiated lightning channels [44, 45].

Several expressions have been considered in the past for the LEMP current waveform: all of them satisfy lightning current waveform parameters which are generally agreed upon [46, 47]. In particular, the empirical equation that is most widely used to reproduce the channel-base currents is the Heidler's function [48, 49], which reads

$i(t)=\frac{I_{01}}{\eta_{1}} \frac{\left(\frac{t}{\tau_{11}}\right)^{n}}{1+\left(\frac{t}{\tau_{11}}\right)^{n}} e^{-\frac{t}{\tau_{21}}}$

where $I_{01}$ is the peak current, $\tau_{11}$ and $\tau_{21}$ are time constants determining current rise-time and decay-time, respectively, $n$ is the current steepness factor, and $\eta_{1}$ is the correction factor of the peak current, given by

$$
\eta_{1}=\exp \left[-\frac{\tau_{11}}{\tau_{21}}\left(\frac{n \tau_{21}}{\tau_{11}}\right)^{\frac{1}{n+1}}\right] \text {. }
$$

Distinguishing between first and subsequent strokes, in this paper we used a single Heidler's function for the first stroke and a sum of two functions like (21) for the subsequent strokes; parameters are given in Table 1.

Other expressions have been also adopted [51], in order to use a unique formula lasting for the whole time period of interest, avoiding the possible time-derivatives discontinuities appearing in the switch between first and subsequent stroke expressions.

\section{Testing and validation of the method}

The accuracy and efficiency of the proposed hybrid method is tested at first comparing its results with the predictions of some analytical formulas for the calculation of resistances to ground valid at DC proposed by the IEEE Standard 142 [52]. Six relevant configurations and their analytical formulas are reported in Table 2. The following assumptions
Table 1 Parameters of the Heidler's functions used to reproduce the channel-base current waveforms (from [50])

Table 2 Analytical formulas of IEEE Std. 142

\begin{tabular}{lllllllll}
\hline Type & $I_{01}(k A)$ & $\tau_{11}(\mu \mathrm{s})$ & $\tau_{21}(\mu \mathrm{s})$ & $n_{1}$ & $I_{02}(\mathrm{kA})$ & $\tau_{12}(\mu \mathrm{s})$ & $\tau_{22}(\mu \mathrm{s})$ & $n_{2}$ \\
\hline First stroke & 28 & 1.8 & 95 & 2 & - & - & - & - \\
Subsequent stroke & 10.7 & 0.25 & 2.5 & 2 & 6.5 & 2.1 & 230 & 2 \\
\hline
\end{tabular}

\begin{tabular}{|c|c|c|}
\hline Ground system & Sketch & DC Ground Resistance \\
\hline Right-angle turn of wire & & $\begin{array}{l}R=\frac{\rho}{4 \pi L} \ln \frac{2 L}{a}+\ln \frac{2 L}{s}-0.2373+0.2146 w+ \\
+0.1035 w^{2}-0.0424 w^{4}\end{array}$ \\
\hline Three-point star & & $\begin{array}{l}R=\frac{\rho}{6 \pi L} \ln \frac{2 L}{a}+\ln \frac{2 L}{s}+1.071-0.209 w+ \\
+0.238 w^{2}-0.054 w^{4}\end{array}$ \\
\hline Four-point star & & $\begin{array}{l}R=\frac{\rho}{8 \pi L} \ln \frac{2 L}{a}+\ln \frac{2 L}{s}+2.912-1.071 w+ \\
+0.645 w^{2}-0.145 w^{4}\end{array}$ \\
\hline Six-point star & & $\begin{array}{l}R=\frac{\rho}{12 \pi L} \ln \frac{2 L}{a}+\ln \frac{2 L}{s}+6.851-3.128 w+ \\
+1.758 w^{2}-0.490 w^{4}\end{array}$ \\
\hline Eight-point star & & $\begin{array}{l}R=\frac{\rho}{16 \pi L} \quad \ln \frac{2 L}{a}+\ln \frac{2 L}{s}+10.98-5.51 w+ \\
+3.26 w^{2}-1.17 w^{4}\end{array}$ \\
\hline \multirow[t]{2}{*}{ Ring of wire } & & $R=\frac{\rho}{2 \pi^{2} D} \ln \frac{4 D}{a}+\ln \frac{4 D}{s}$ \\
\hline & & $\begin{array}{l}\text { Length of arm } L \text {, depth } s / 2 \text {, wire radius } a, \\
\text { diameter of ring } D \text {, and } w=\frac{s}{L}\end{array}$ \\
\hline
\end{tabular}


Table 3 Comparison between numerical results and predictions of analytical formulas (IEEE Std. 142) under DC conditions

\begin{tabular}{lll}
\hline Ground system & Numerical method & Analytical formula \\
\hline Right-angle turn & 8.41 & 8.43 \\
Three-point star & 6.45 & 6.48 \\
Four-point star & 5.50 & 5.54 \\
Six-point star & 4.61 & 4.66 \\
Eight-point star & 4.19 & 4.24 \\
Ring of wire & 3.49 & 3.53 \\
\hline
\end{tabular}

have been considered: ground resistivity $\rho=100 \Omega \times \mathrm{m}$, length of arm $L=10 \mathrm{~m}$, depth $s=0.8 \mathrm{~m}$, wire radius $a=$ $3.5 \mathrm{~mm}$.

The comparison among the results of the hybrid method and the predictions of the analytical formulas is reported in Table 3, considering a unit injected current. Moreover, the
3D maps of the potential on the ground are reported in Fig. 4. Excellent agrement between the numerical results and the predictions through analytical formulas is shown in each of the examined case.

Successively, the proposed approach has been applied to classical transient test cases to demonstrate the effectiveness of the procedure and to simultaneously asses the influence of the dominant parameters on the dynamic behavior of grounding electrodes under lightning strokes.

The considered test cases are two and are taken from [53]; they are based on the following configurations:

- horizontal copper-made electrode with length $30 \mathrm{~m}$ (denoted as long electrode) and diameter $1.4 \mathrm{~cm}$, buried at a depth of $0.8 \mathrm{~m}$ in a resistive soil with $\varepsilon_{\mathrm{r}, \mathrm{g}}=10$ and $\rho_{\mathrm{g}}=300 \Omega \mathrm{m}$, whose results are reported in Fig. 5;

- horizontal copper-made electrode with length $3 \mathrm{~m}$ (denoted as short electrode) and diameter $1.4 \mathrm{~cm}$, buried

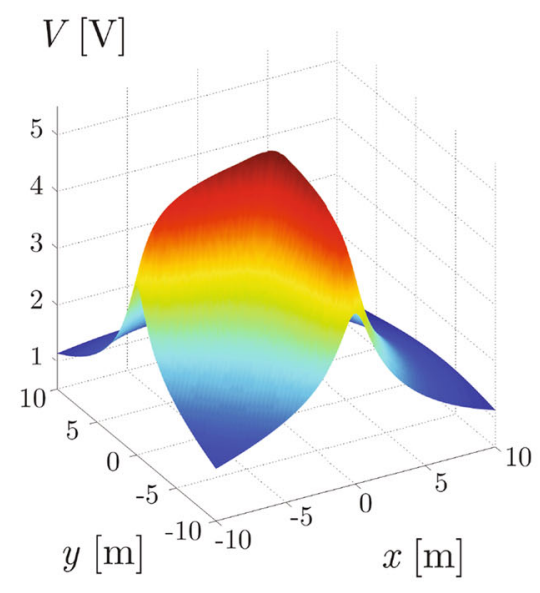

(a) Right-angle turn

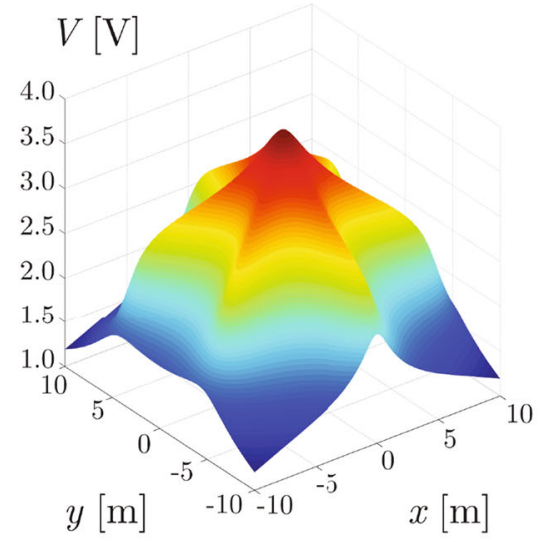

(d) Six-point star

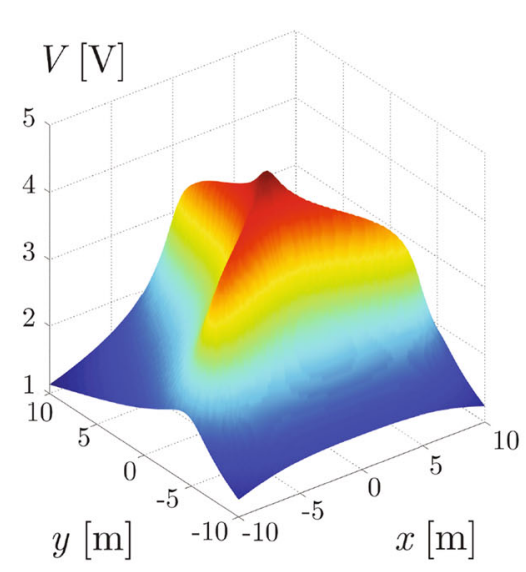

(b) Three-point star

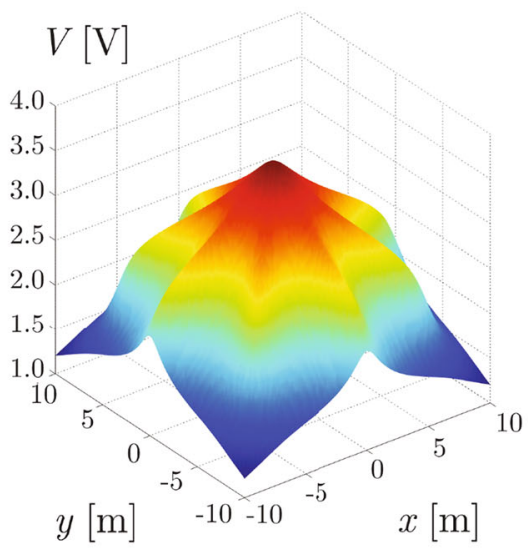

(e) Eight-point star

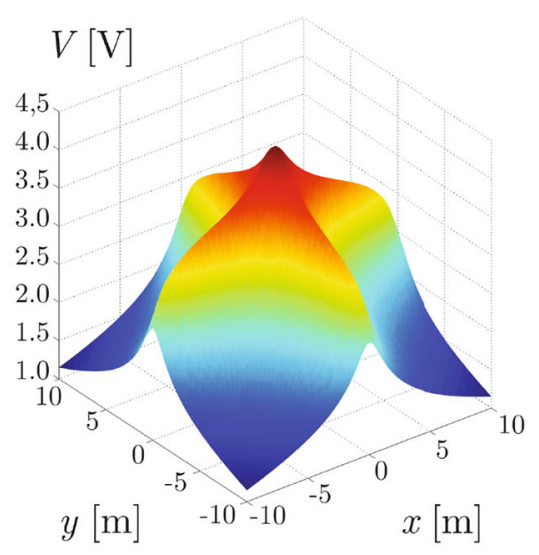

(c) Four-point star

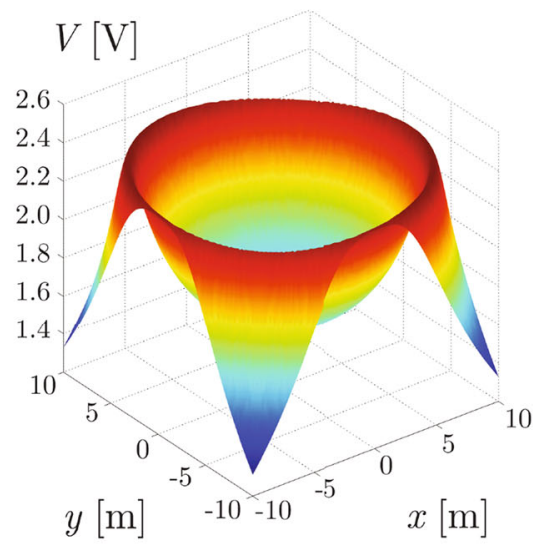

(f) Ring of wire

Fig. 4 Potential distribution on the earth surface for different ground system geometries buried at a depth of $0.8 \mathrm{~m}$. a Right-angle turn, $\mathbf{b}$ threepoint star, $\mathbf{c}$ four-point star, $\mathbf{d}$ six-point star, e eight-point star, $\mathbf{f}$ ring of wire 


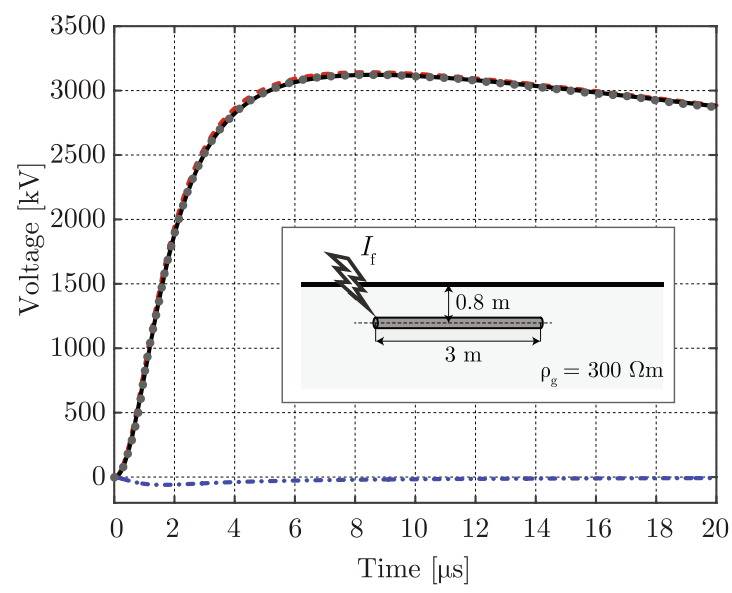

(a) First stroke

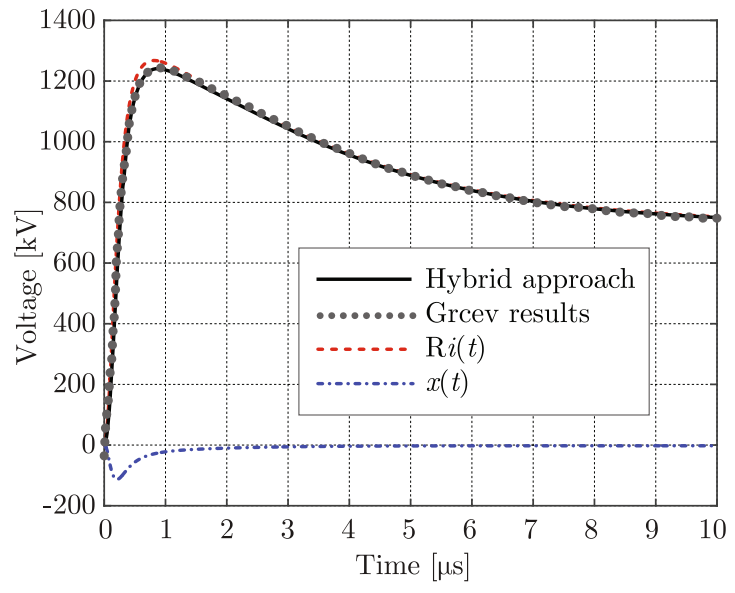

(b) Subsequent stroke

Fig. 5 Computed results for a resistive soil $\left(\rho_{\mathrm{g}}=300 \Omega \mathrm{m}\right)$ and a short electrode $(L=3 \mathrm{~m})$. The DC resistance is $R=105.54 \Omega$. a First stroke, b subsequent stroke

at a depth of $0.8 \mathrm{~m}$ in a conductive soil with $\varepsilon_{\mathrm{r}, \mathrm{g}}=10$ and $\rho_{\mathrm{g}}=300 \Omega \mathrm{m}$, whose results are reported in Fig. 6 .

In each figure the transient potential $v(t)$ is reported, computed by means of the proposed approach under the current pulse $i(t)$ of a first or subsequent return stroke whose parameters are reported in Table 1 . The transient voltage is subdivided into two terms as proposed in [53]

$v(t)=R i(t)+x(t)$,

where $R$ is the DC grounding resistance. The first term in (23), named resistive component of the voltage drop, approximates the linear resistive voltage drop in the grounding system, while the second term, named reactive component of the voltage drop, accounts for the frequencydependent phenomena, i.e., the inductive and capacitive couplings between the elements of the grounding system.

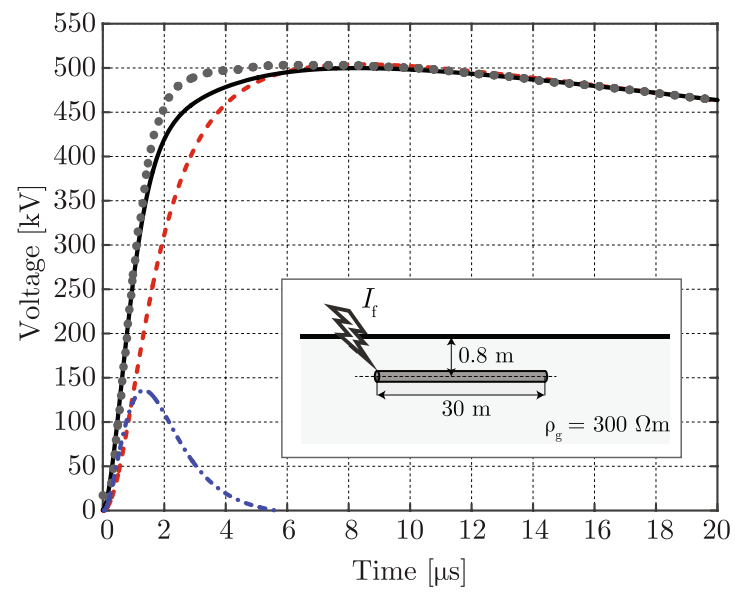

(a) First stroke
The comparison between the rise voltage predictions of the two proposed methods for all the considered cases shows that the hybrid approach tends to estimate lower picks and higher rise times than the Grcev [53] results, based on a electromagnetic full-wave approach: the hybrid approach tends to estimate higher capacitive couplings between the elements of the grounding system, flattening the surge front and reducing the voltage picks.

\section{Results}

Even if most of the wind turbines manufacturers specify the grounding system configurations for their own towers, a typical wind turbine earthing system arrangement is shown in Fig. 7 [5]. It mainly consists of a square of galvanized steel flanges (gray line) buried at $2 \mathrm{~m}$ depth, two copper

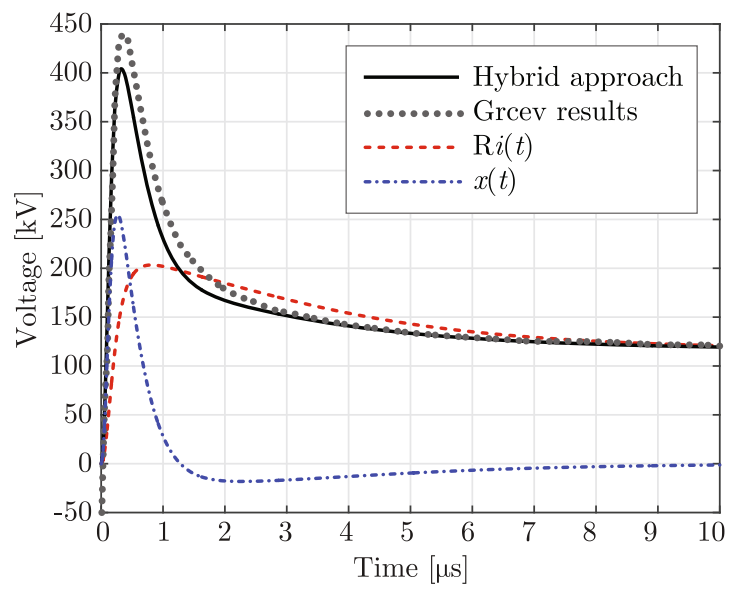

(b) Subsequent stroke

Fig. 6 Computed results for a resistive soil $\left(\rho_{\mathrm{g}}=300 \Omega \mathrm{m}\right)$ and a long electrode $(L=30 \mathrm{~m})$. The DC resistance is $R=16.93 \Omega$. a First stroke, b subsequent stroke 
Fig. 7 Typical wind turbine grounding system arrangement: a prospective view; $\mathbf{b}$ front view

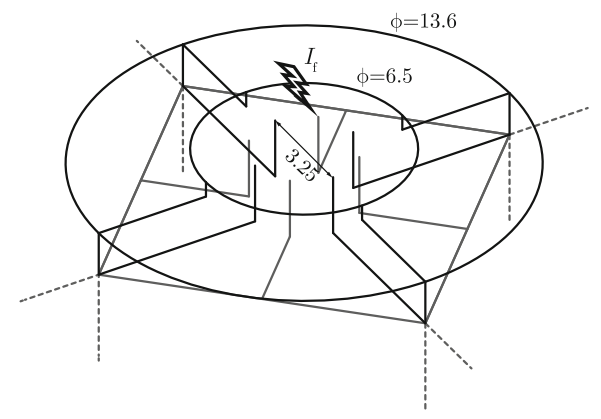

(a)

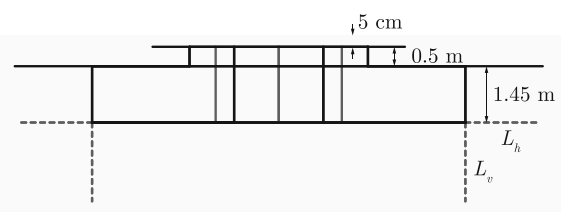

(b)

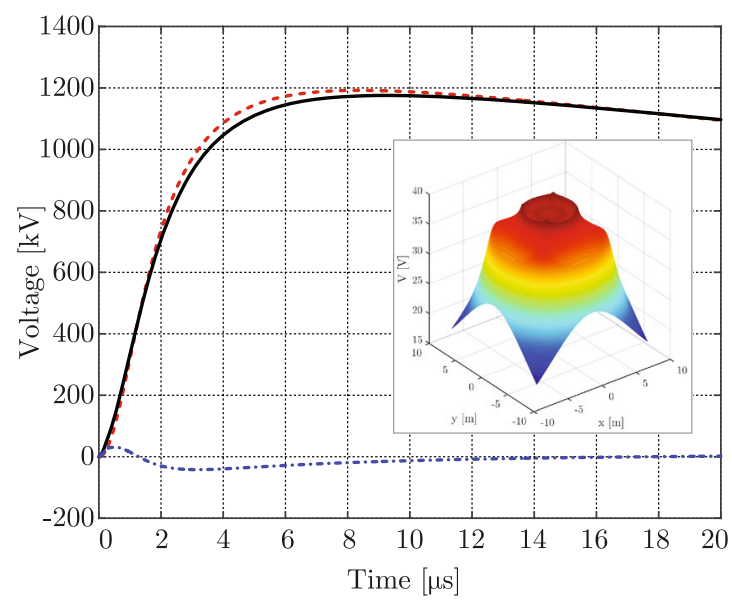

(a) First stroke

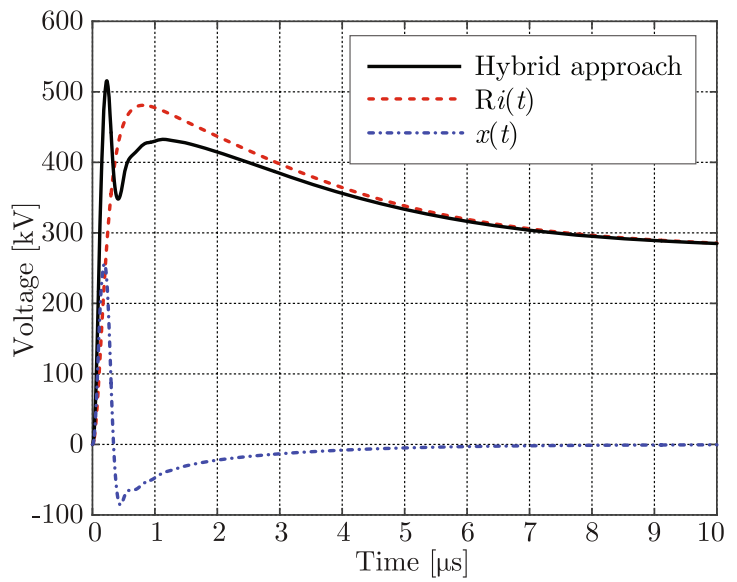

(b) Subsequent stroke

Fig. 8 Computed results for the wind grounding system without additional horizontal or vertical electrodes. The inset in a shows the DC potential map on the ground when the injected current is $1 \mathrm{~A}$. The DC resistance is $R=40.04 \Omega$. a First stroke, b subsequent stroke

ring wires (black line) at two different levels, the smaller one at $5 \mathrm{~cm}$ depth while the larger one at $55 \mathrm{~cm}$ depth, four wires connecting the inner circle with the outer one, and additional eight copper wires connecting the outer circle and the square with the tower. Additional horizontal and vertical electrodes, respectively of length $L_{\mathrm{h}}$ and $L_{\mathrm{v}}$, may be added to the ground system in order to obtain prescribed values of the ground resistance.

The grounding system is placed in a homogenous soil of low conductivity, $\rho_{\mathrm{g}}=1200 \Omega \mathrm{m}$, which is a realistic scenario for typical wind farms. The relative permittivity is assumed to be $\varepsilon_{\mathrm{r}}=9$.

Figure 8 show the transient potential of the basic grounding system without any additional vertical or horizontal electrode, under a first or subsequent stroke. The inset of Fig. 8a shows the potential distribution on the ground at DC when the injected current is equal to $1 \mathrm{~A}$. It can be seen in Fig. 8a that the ground system shows a resistive behavior under the first stroke, but when a subsequent stroke is considered, which is characterized by a fast rising front with higher frequency content (up to 10
$\mathrm{MHz}$ ), it can be observed in Fig. 8b that the reactive component of the voltage drop plays a paramount role in the initial transient response, giving rise to a voltage peak that is higher than the peak predicted by the resistive response only.

Successively, we investigated the influence of additional vertical electrodes on the transient behavior of the grounding system. The basic earthing system has been upgraded with four vertical electrodes of variable length $L_{\mathrm{v}}$ as shown in Fig. 7. Results reported in Fig. 9 show that additional short electrodes ( 3 or $5 \mathrm{~m}$ long) do not significantly decrease the transient response of the grounding system, under either a first or a subsequent stroke, with respect to the basic case. In order to clearly decrease the transient voltage it is necessary to resort to relatively long electrodes, e.g., with lengths ranging between 10 and $15 \mathrm{~m}$.

Then we assessed the effect of horizontal electrodes of various length $L_{\mathrm{h}}$; the grounding system has been upgraded with four horizontal electrodes connected at the four vertices and buried at $2 \mathrm{~m}$ depth as shown in Fig. 7. Figure 10 show the transient voltage raise of the grounding system at 


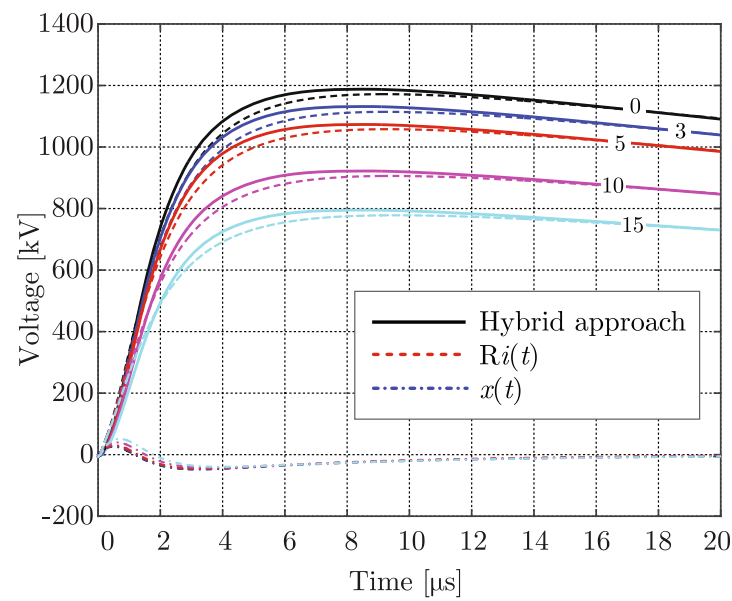

(a) First stroke

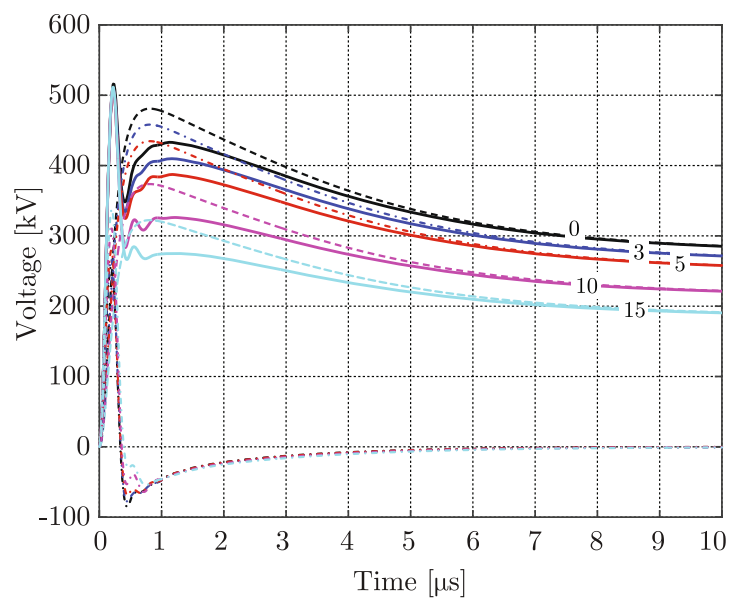

(b) Subsequent stroke

Fig. 9 Computed results for the wind grounding system with additional vertical electrodes of different lengths $L_{\mathrm{v}}$. The DC resistances are $R_{3 \mathrm{~m}}=38.15 \Omega, R_{5 \mathrm{~m}}=36.19 \Omega, R_{10 \mathrm{~m}}=31.11 \Omega$, and $R_{15 \mathrm{~m}}=26.84 \Omega$. a First stroke, b subsequent stroke

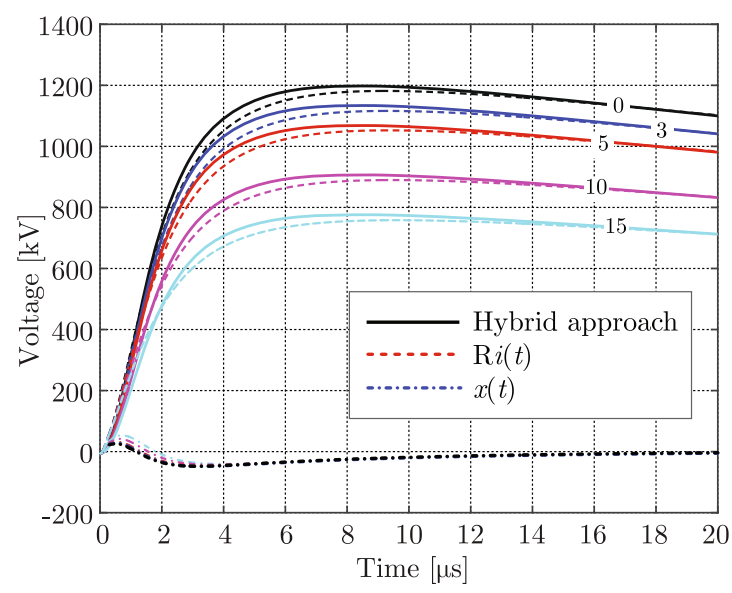

(a) First stroke

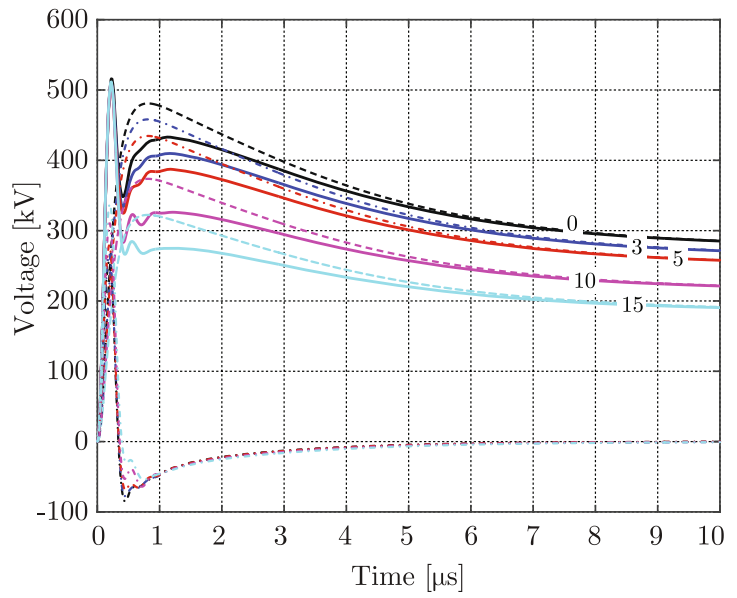

(b) Subsequent stroke

Fig. 10 Computed results for the wind grounding system with additional horizontal electrodes of different lengths $L_{\mathrm{h}}$. The DC resistances are $R_{3 \mathrm{~m}}=37.91 \Omega, R_{5 \mathrm{~m}}=35.73 \Omega, R_{10 \mathrm{~m}}=33.35 \Omega$, and $R_{15 \mathrm{~m}}=26.01 \Omega$. a First stroke, $\mathbf{b}$ subsequent stroke

the feeding point. Compared to the case of vertical electrodes, these results clearly show that there is no appreciable difference between the vertical and horizontal case, leading to the conclusion that the horizontal case is preferable because placing horizontal electrodes is usually cheaper especially in rocky terrains. It should also be observed that the computed DC resistances for the case of horizontal electrodes are slightly lower than the corresponding resistances in the case of vertical electrodes; this observation corroborates that horizontal electrodes are preferable.

To conclude the discussion of the results, a last remark is necessary about the protection level ensured by the earthing system according to international standards such as the IEC 62305 [54]. The earthing system usually comprises a single integrated structure that is suitable for several purposes, i.e., lightning protection, power and telecommunication systems earthing. The standard recommends that in dealing with the dispersion of the lightning current into the ground, the shape and dimensions of the earthing system are the important criteria to minimize any potentially dangerous overvoltages. In general, a low earthing resistance at industrial frequency is recommended, usually below $10 \Omega$. The standard, in the part number three [54, Section 5.4], suggests operative formulas for two basic types of earth electrode arrangements: type-A that comprises horizontal or vertical earth electrodes installed outside the structure and type-B that comprises either a ring conductor external to the structure to be protected, in contact with the soil for at least $80 \%$ of its total length, or 
a foundation earth electrode forming a closed loop. The Type-B clearly apply to the present case under analysis. In this case the standard prescribes that for the ring earth electrode (or foundation earth electrode), the mean radius $r_{\mathrm{e}}$ of the area enclosed by the ring earth/fundation electrode shall be not less than a prescribed value $l_{1}$ which depends on the lightning protection system (LPS) class and on the resistivity of the soil $\rho_{\mathrm{g}}$. In our case, with $\rho_{\mathrm{g}}=1200 \Omega \mathrm{m}$, the prescribed values $l_{1}$ are $27 \mathrm{~m}$ for Class I, $14 \mathrm{~m}$ for Class II, $5 \mathrm{~m}$ for Class III and IV. In our case, considering only the outer ring of diameter $13.6 \mathrm{~m}\left(r_{\mathrm{e}}=6.8 \mathrm{~m}\right.$, the Class should be III. Anyway, the standard adds that when the required value of $l_{1}$ is larger than the convenient value of $r_{\mathrm{e}}$, additional horizontal or vertical electrodes shall be added with individual lengths $l_{\mathrm{h}}=l_{1}-r_{\mathrm{e}}$ and $l_{\mathrm{v}}=\left(l_{1}-r_{\mathrm{e}}\right) / 2$. The standard recommends that the number of electrodes shall be not less than the number of the down-conductors, with a minimum of two. Hence, adding horizontal electrodes of length $15 \mathrm{~m}$, we could obtain the protection Class II while adding vertical electrodes of the same length, we could reach Class I according to the standard. It is interesting to note that the overperformance claimed by the standard of the vertical electrodes on the horizontal ones is not observed on the computed results. In order to better investigate this issue, we have repeated the simulations changing the value of the grounding resistivity $\rho_{\mathrm{g}}$ between 30 and $3000 \Omega \mathrm{m}$, and we have always observed that the performance of vertical and horizontal electrodes of the same length is almost the same.

\section{Conclusions}

The suitability of a new hybrid approach for the computation of the behavior of ground systems in the frequency domain has been investigated. The formulation is based on circuit theory and method of moments, to fully account for resistive, inductive and capacitive couplings, and the analysis is carried out in the frequency domain. When needed, the corresponding transient response is obtained by means of the inverse Fourier transform. The method has been used to characterize the transient behavior of wind turbine grounding systems in several configurations, investigating the relevant aspects described in International Standards.

Open Access This article is distributed under the terms of the Creative Commons Attribution 4.0 International License (http://crea tivecommons.org/licenses/by/4.0/), which permits unrestricted use, distribution, and reproduction in any medium, provided you give appropriate credit to the original author(s) and the source, provide a link to the Creative Commons license, and indicate if changes were made.

\section{References}

1. Araneo, R., Grasselli, U., Celozzi, S.: Assessment of a practical model to estimate the cell temperature of a photovoltaic module. Int. J. Energy Environ. Eng. 5, 1-15 (2014)

2. Lucchetti, E., Barbier, J., Araneo, R.: Assessment of the technical usable potential of the TUM shaft hydro power plant on the Aurino River, Italy. Renew. Energy 60, 648-654 (2013)

3. Araneo, R., Celozzi, S., Lovat, G.: Shielding effectiveness of artificial magnetic screens in the VHF band. In: Proc. IEEE Electromagn. Compat. Symp., 17-21 Aug, pp. 72-77 (2009)

4. Rodrigues, R., Mendes, V., Catalão, J.: Protection of wind energy systems against the indirect effects of lightning. Renew. Energy 36(11), 2888-2896 (2011)

5. Cavka, D., Poljak, D., Doric, V., Goic, R.: Transient analysis of grounding systems for wind turbines. Renew. Energy 43, 284-291 (2012)

6. Napolitano, F., Paolone, M., Borghetti, A., Nucci, C.A., Cristofolini, A., Mazzetti, C., Fiamingo, F., Marzinotto, M.: Models of wind-turbine main shaft bearings for the development of specific lightning protection systems. IEEE Trans. Electromagn. Compat. 53(1), 99-107 (2007)

7. Calixto, W., Neto, L., Wu, M., Yamanaka, K., da Paz Moreira,, E.: Parameters estimation of a horizontal multilayer soil using genetic algorithm. IEEE Trans. Power Deliv. 25(3), 1250-1257 (2010)

8. Yamamoto, K., Yanagawa, S., Yamabuki, K., Sekioka, S., Yokoyama, S.: Analytical surveys of transient and frequencydependent grounding characteristics of a wind turbine generator system on the basis of field tests. IEEE Trans. Power Deliv. 25(4), 3035-3043 (2010)

9. Grcev, L.D., Heimbach, M.: Frequency dependent and transient characteristics of substation grounding systems. IEEE Trans. Power Deliv. 12(1), 172-178 (1997)

10. Visacro, S., Soares, A.: HEM: A model for simulation of lightning-related engineering problems. IEEE Trans. Power Deliv. 20(2), 1206-1208 (2005)

11. Cortina, R., Pioltini, G., Celozzi, S., D’Amore, M.: Telecommunication systems on power distribution networks: high frequency performances of carrier channels. IEEE Trans. Power Deliv. 9(2), 654-660 (1994)

12. Li, Z.-X., Yin, Y., Zhang, C.-X., Zhang, L.-C.: A mathematical model for the transient lightning response from grounding systems. Prog. Electromagn. Res. B 57, 47-61 (2014)

13. Li, Z.X., Li, G.F., Fan, J.B., Yin, Y.: Quasi-static complex image method for a current point source in horizontally stratified multilayered earth. Prog. Electromagn. Res. B 34, 187-204 (2011)

14. Liu, Y., Theethayi, N., Thottappillil, R.: An engineering model for transient analysis of grounding system under lightning strikes: nonuniform transmission-line approach. IEEE Trans. Power Deliv. 20(2), 722-730 (2005)

15. Liu, Y., Zitnik, M., Thottappillil, R.: An improved transmissionline model of grounding system. IEEE Trans. Electromagn. Compat. 43(3), 348-355 (2001)

16. Theethayi, N., Baba, Y., Rachidi, F., Thottappillil, R.: On the choice between transmission line equations and full-wave maxwell's equations for transient analysis of buried wires. IEEE Trans. Electromagn. Compat. 50(2), 347-357 (2008)

17. Theethayi, N., Thottappillil, R., Paolone, M., Nucci, C.A., Rachidi, F.: External impedance and admittance of buried horizontal wires for transient studies using transmission line analysis. IEEE Trans. Dielect. Elect. Insulation 14(3), 751-761 (2007)

18. Li, Z., Fan, J.: Numerical calculation of grounding system in low frequency domain based on the boundary element method. Int. J. Numer. Methods Eng. 73, 685-705 (2008) 
19. Li, Z., Fan, J., Chen, W.: Numerical simulation of substation grounding grids buried in both horizontal and vertical multilayer earth model. Int. J. Numer. Methods Eng. 69, 2359-2380 (2007)

20. Li, Z.-X., Chen, W.-J.: Numerical simulation grounding system buried within horizontal multilayer earth in frequency domain. Int. J. Numer. Methods Eng. 23, 11-27 (2007)

21. Li, Z., Chen, W., Fan, J., Lu, J.: A novel mathematical modeling of grounding system buried in multilayer earth. IEEE Trans. Power Deliv. 21(3), 1267-1272 (2006)

22. Araneo, R., Celozzi, S.: Extraction of equivalent lumped circuits of discontinuities using the finite-difference time-domain method. Proc. IEEE Electromagn. Compat. Symp. 1, 119-122 (2002)

23. Araneo, R., Dughiero, F., Fabbri, M., Forzan, M., Geri, A., Morandi, A., Lupi, S., Ribani, P., Veca, G.: Electromagnetic and thermal analysis of the induction heating of aluminum billets rotating in DC magnetic field. COMPEL 27(2), 467-479 (2008)

24. Celozzi, S., D'Amore, M.: Magnetic field attenuation of nonlinear shields. IEEE Trans. Electromagn. Compat. 38(3), 318-326 (1996)

25. Duong, M.Q., Grimaccia, F., Leva, S., Mussetta, M., Le, K.H.: Improving transient stability in a grid-connected squirrel-cage induction generator wind turbine system using a fuzzy logic controller. Energies 8(7), 6328-6349 (2015)

26. Zhuang, L., Zhang, Y., Hu, W., Yu, W., Zhu, G.-Q.: Automatic incorporation of surface wave poles in discrete complex image method. Prog. Electromagn. Res. 80, 161-178 (2008)

27. Li, Z.-X., Shi, W.-D., Yin, Y.: Transient lightning response of grounding grid buried in multilayered earth model based on dynamic sate complex image method in time domain. In: Power System Technology (POWERCON), 2014 International Conference on IEEE, pp. 1635-1643 (2014)

28. Li, Z.-X., Yin, Y., Zhang, C.-X., Zhang, L.-C.: Numerical simulation of currents distribution along grounding grid buried in horizontal multi-layered earth in frequency domain based dynamic state complex image method. Electr. Power Compon. Syst. 43(14), 1573-1582 (2015)

29. Otero, A.F., Cidras, J., Alamo, J.L.: Frequency-dependent grounding system calculation by means of a conventional nodal analysis technique. IEEE Trans. Power Deliv. 14(3), 873-878 (1999)

30. Araneo, R., Lovat, G., Celozzi, S.: Shielding effectiveness of periodic screens against finite high-impedance near-field sources. IEEE Trans. Electromagn. Compat. 53(3), 706-716 (2011)

31. Araneo, R., Wang, C., Gu, X., Drewniak, J., Celozzi, S.: Efficient modeling of discontinuities and dispersive media in printed transmission lines. IEEE Trans. Magn. 38(2), 765-768 (2002)

32. Araneo, R., Lovat, G.: Analysis of the shielding effectiveness of metallic enclosures excited by internal sources through an efficient Method of Moment approach. ACES J. 25(7), 600-611 (2010)

33. Kourkoulos, V., Cangellaris, A.C.: Accurate approximation of Green's functions in planar stratified media in terms of a finite sum of spherical and cylindrical waves. IEEE Trans. Antennas Propag. 54(5), 1568-1576 (2006)

34. Michalski, K.A., Mosig, J.R.: Multilayered media Green's functions in integral equation formulations. IEEE Trans. Antennas Propag. 45(3), 508-519 (1997)

35. Lovat, G., Araneo, R., Celozzi, S.: Dipole excitation of periodic metallic structures. IEEE Trans. Antennas Propag. 59(6), 2178-2189 (2011)

36. Polimeridis, A.G., Mosig, J.R.: Fast computation of Sommerfeld integral tails via direct integration based on double exponentialtype quadrature formulas. IEEE Trans. Antennas Propag. 59(2), 694-699 (2011)
37. Wilton, D., Champagne, N.: Evaluation and integration of the thin wire kernel. IEEE Trans. Antennas Propag. 54(4), 1200-1206 (2006)

38. Resende, U., Moreira, M., Afonso, M.: Evaluation of singular integral equation in MoM analysis of arbitrary thin wire structures. IEEE Trans. Magn. 50(2), 457-460 (2014)

39. Ma, J., Rokhlin, V., Wandzura, S.: Generalized Gaussian quadrature rules for systems of arbitrary functions. SIAM J. Numer. Anal. 33(3), 971-996 (1996)

40. Romero, C., Rachidi, F., Paolone, M., Rubinstein, M.: Statistical distributions of lightning currents associated with upward-negative flashes based on the data collected at the Santis Tower in 2010 and 2011. IEEE Trans. Power Deliv. 28(3), 1804-1812 (2013)

41. Diendorfer, G., Pichler, H., Mair, M.: Some parameters of negative upward initiated lightning to the Gaisberg Tower (2000-2007). IEEE Trans. Electromagn. Compat. 51(3), 443-452 (2009)

42. Visacro, S., Soares, J.A., Schroeder, M.A.O., Cherchiglia, L.C.L., de Sousa, V.J.: Statistical analysis of lightning current parameters: measurements at Morro do Cachimbo station. J. Geophys. Res. 109(D01105), 1-11 (2004)

43. Heidler, F., Wiesinger, J., Zischank, W.: Lightning currents measured at a telecommunication tower from 1992 to 1998. In: 14th International Zurich symposium on electromagnetic compatibility, Zurich, p. 6 (2001)

44. Rakov, V.A., Uman, M.A., Wang, D., Rambo, K.J., Crawford, D.E., Schnetzer, G.H.: Lightning properties from triggeredlightning experiments at Camp Blanding, Florida (1997-1999). In: 25th ICLP (International conference on lightning protection), Rhodes, pp. 54-59 (2000)

45. Rakov, V., Uman, M.A.: Lightning: physics and effects. Cambridge University Press (2003)

46. Rakov, V.A.: Lightning parameters for engineering applications. In: Asia-Pacific Symposium on Electromagnetic Compatibility (APEMC). IEEE, Beijing, pp. 1120-1123 (2013)

47. IEEE Std. 1410: Guide for improving the lightning performance of electric power overhead distribution lines. IEEE, pp. 1-73 (2011). doi:10.1109/IEEESTD.2011.5706451

48. Heidler, F.: Analytische blitzstromfunktion zur LEMP-berechnung. In: Proceedings of the 18th Int. conf. on lightning protection, Munich, September 16-20 1985, pp. 63-66 (1985)

49. Araneo, R., Celozzi, S., Tatematsu, A., Rachidi, F.: Time-domain analysis of building shielding against lightning electromagnetic fields. IEEE Trans. Electromagn. Compat. 57(3), 397-404 (2015)

50. Rachidi, F., Janischewskyj, W., Hussein, A.M., Nucci, C.A., Guerrieri, S., Kordi, B., Chang, J.-S.: Current and electromagnetic field associated with lightning return strokes to tall towers. IEEE Trans. Electromagn. Compat. 43(3), 356-367 (2001)

51. Conti, A.D., Visacro, S.: Analytical representation of single- and double-peaked lightning current waveforms. IEEE Trans. Electromagn. Compat. 49(2), 448-451 (2007)

52. IEEE Std. 142: IEEE recommended practice for grounding of industrial and commercial power systems-redline. IEEE, pp. 1-215 (1982)

53. Grcev, L.: Time- and frequency-dependent lightning surge characteristics of grounding electrodes. IEEE Trans. Power Deliv. 24(4), 2186-2196 (2009)

54. IEC Std. 62305-3: Protection against lightning-part 3: physical damage to structures and life hazard. IEC Std (2006) 